Journal of Patient-Centered

Volume 2

Issue 4 - Integrative Medicine

Article 17

$11-20-2015$

\title{
Prognostic Indices for Hospitalized Older Adults: A Meta-Analysis and Systematic Review
}

Ariba Khan

Ayesha Maria

James Hocker

Maharaj Singh

Michelle Simpson

Follow this and additional works at: https://aah.org/jpcrr

Part of the Geriatrics Commons, and the Health Information Technology Commons

\section{Recommended Citation}

Khan A, Maria A, Hocker J, Singh M, Simpson M. Prognostic Indices for Hospitalized Older Adults: A MetaAnalysis and Systematic Review. J Patient Cent Res Rev 2015;2:206-207. http://dx.doi.org/10.17294/ 2330-0698.1227

Published quarterly by Midwest-based health system Advocate Aurora Health and indexed in PubMed Central, the Journal of Patient-Centered Research and Reviews (JPCRR) is an open access, peer-reviewed medical journal focused on disseminating scholarly works devoted to improving patient-centered care practices, health outcomes, and the patient experience. 
Purpose: To explore the geographic distribution and associated risk factors for maternal GBS colonization and infant death prior to discharge in eastern Wisconsin births.

Methods: Retrospective study of institutional data from PeriData.net, a comprehensive birth registry, utilizing data from 2007 through 2013 at all Aurora medical centers. Categorical variables were analyzed with chi-square tests, and ordinal or continuous variables by Mann-Whitney or two-sample t-tests. Binary regression was used for multivariate modeling.

Results: Population demographics $(\mathrm{N}=99,305)$ were mean age 28 years, 59\% married, 64\% white, $42 \%$ governmentinsured, 39\% nulliparous, mean prepregnancy body mass index (BMI) of 27, gestational age of 39 weeks, birth weight of 3,296 $\mathrm{g}$ and $26 \% \mathrm{C}$-section rate. The GBS colonization rate was 22.3\%. Among ZIP codes with > 100 subjects, 8 ZIP codes had a GBS-positive rate $>30 \%$ (7 in Milwaukee, 1 in Kohler). GBS colonization was higher in blacks (34\%) than whites $(20 \% ; \mathrm{P}<0.0001)$, in unmarried women $(26 \%$ vs. $20 \%$; $\mathrm{P}<0.0001$ ), with increasing BMI (mean BMI 27.3 if GBS-positive vs. 26.6; $\mathrm{P}<0.0001$ ) and based on ZIP code group $(\mathrm{P}<0.0001)$; and was predictive of neonatal antibiotics for sepsis $(26 \%$ if GBS-positive vs. $22 \%$; $<<0.0001)$. In multivariate analysis, unmarried status, higher BMI, race and ZIP code were predictive of GBS colonization. Rate of infant death during birth hospitalization was $0.57 \%(n=558)$ and varied by ZIP code group. GBS colonization was negatively associated with infant death $(0.25 \%$ in GBS-positive vs. $0.66 \% ; \mathrm{P}<0.0001 ; \mathrm{N}=98,065$ with lethal anomalies and stillbirths excluded). This association remained when controlling for gestational age. In multivariate analysis, death rate was associated with one ZIP code group, no prenatal care, preterm labor, vaginal bleeding, hydramnios, oligohydramnios, lower gestational age and maternal GBS (negative predictor).

Conclusion: Geographic characteristics are associated with infant death during birth hospitalization and maternal GBS colonization. Demographic characteristics are only associated with maternal GBS colonization. It is unclear if maternal GBS colonization is "protective" against infant demise due to increased surveillance.

\section{An Automated Model Using Electronic Health Record Data to Identify Delirium Among Hospitalized Older Adults: A Pilot Project}

Ariba Khan, Maharaj Singh, Hina Singh, Ayesha Maria, Michelle Simpson

\section{Department of Geriatrics, Aurora UW Medical Group and Aurora Sinai Medical Center; Aurora Research Institute, Aurora Health Care}

Background: Delirium is a serious change in mental status with adverse outcomes, but remains underrecognized. The electronic health record (EHR) may assist in the identification of delirium.

Purpose: This study was performed to generate an automated delirium identification model using data from the EHR among hospitalized older adults.

Methods: Inpatients 65 years and older were included in this cross-sectional study. The researchers used "confusion assessment method" as the gold standard to identify delirium. Four categories of variables were obtained from the EHR on the day of and the day prior to researcher assessment: 1) hypoactive delirium (any one of the following: nurse's assessment of motor retardation or reduced level of consciousness or decline in activities of daily living [ADL] score); 2) hyperactive delirium (any one of the following: use of restraints or antipsychotic medications or nurse's assessment noting a change in mental status or poor attention or motor agitation or poor thought process or anxiety); 3) patient factors (any one of the following: dementia, age, mean blood urea nitrogen and serum creatinine); and 4) health care-associated factors (any one of the following: urinary catheter, surgical procedure, brain imaging). Relationships were analyzed using chi-square or Fisher's test as appropriate. Statistical significance was set at $\mathrm{P}<0.05$. Results: Ninety-two participants in three hospitals were included in the analysis. Of these, mean age was $77 \pm 8.8$ years and $54 \%$ were female, $70 \%$ had a Morse fall score $>45$, and mean ADL score was 10 of 12 . The prevalence of delirium was $17 \%$. In the univariate analysis, variables associated with delirium included abnormal mental status ( $94 \%$ vs. $41 \%$; $\mathrm{P}<0.0001$ ); reduced level of consciousness $(69 \%$ vs. $9 \% ; \mathrm{P}<0.0001)$, motor retardation $(50 \%$ vs. $13 \% ; \mathrm{P}<0.0007)$, motor agitation $(38 \%$ vs. $7 \% ; \mathrm{P}=0.004)$ abnormal attention $(81 \%$ vs. $12 \%$; $\mathrm{P}<0.0001)$, abnormal thought process (56\% vs. $11 \%$; $\mathrm{P}<0.001)$, dementia $(31 \%$ vs. $11 \% ; \mathrm{P}=0.03$ ), age ( 82 vs. 72 years; $\mathrm{P}=0.02$ ), number of medications ( 10 vs. $12 ; \mathrm{P}=0.0313$ ), use of antipsychotic medication ( $31 \%$ vs. $7 \%$; $\mathrm{P}=0.004)$, mean Braden score $(15$ vs. $18 ; \mathrm{P}=0.0038)$ and Morse fall score $>45(94 \%$ vs. $59 \% ; \mathrm{P}=0.02)$. In the multivariate analysis, factors associated with delirium included reduced level of consciousness and abnormal attention (area under curve 0.920).

Conclusion: This pilot study demonstrates that variables present in the EHR may be used to develop an automated model to identify delirium in hospitalized older adults. These findings need to be validated in a larger study and define if the model performs well in predicting clinical outcomes.

\section{Prognostic Indices for Hospitalized Older Adults: A Meta-Analysis and Systematic Review}

Ariba Khan, Ayesha Maria, James Hocker, Maharaj Singh, Michelle Simpson

Department of Geriatrics, Aurora UW Medical Group and Aurora Sinai Medical Center; Aurora Research Institute, Aurora Health Care

Background: A prognostication predictive model incorporated into the electronic health record (EHR) may be useful in assisting the health care team in accurately predicting mortality and may be used in appropriately 
allocating palliative care services.

Purpose: To systematically review and summarize current medical literature regarding the factors predictive of mortality in an inpatient population above 65 years of age.

Methods: Nondisease-specific prognostication indices that predict 1-year mortality in an inpatient population of adults over age 65 were included. We excluded studies that estimated intensive care unit, disease-specific or in-hospital mortality. A MEDLINE, CINAHL, Ovid and Cochrane literature search of English-language articles that developed and/or validated a prognostication index to predict mortality was performed. Review of 3,600 citations revealed 53 articles that reported variables associated with mortality. Based on the inclusion criteria, 9 studies were included in the final analysis. Data was extracted from the 9 studies using the following parameters: adequate method of description of population, nonbiased selection of patients, low loss to follow-up, adequate prognostic factor measurements, adequate outcome measurements and methods of validation. We performed qualitative analysis on 5 studies and 4 studies were pooled for a quantitative meta-analysis.

Results: The 1-year mortality rate for the 21,338 patients included in all the studies was 31\% (95\% confidence interval [CI]: 31.3-32.6); mean age was 80.6 years. Factors significantly associated with mortality included male sex (odds ratio [OR]: $1.25,95 \%$ CI: 1.09-1.42; $\mathrm{P}<0.001$ ), congestive heart failure (OR: $0.41,95 \% \mathrm{CI}$ : 0.37-0.45; $\mathrm{P}<0.001$ ), chronic obstructive pulmonary disease (OR: 3.2, 95\% CI: $0.42-24.9 ; \mathrm{P}=0.26)$, myocardial infarction (proportion $0.39 ; \mathrm{P}<0.001$ ), and cerebrovascular disease (proportion 0.38, 95\% CI: 0.32-0.44; $\mathrm{P}<0.001$ ).

Conclusion: One-year mortality for inpatients aged > 65 years was high and associated with male sex, chronic obstructive pulmonary disease and congestive heart failure. Generalization of these findings to all older adults should be made with caution because of insufficient published information. In the future, our results may be used to develop a prognostication tool that draws patient data in real time from the EHR to identify vulnerable older adults in the hospital with end-of-life needs.

\section{Chronic Illness Management in Teams of Urban Multidisciplinary Scholars (CIMTUMS) - Part II}

John R. Brill, Diane Ames, Christine B. Groth, Helen Yu

Academic Affairs, Aurora UW Medical Group; Concordia University; Pharmacy, Aurora Health Care; University of Wisconsin School of Medicine and Public Health

Background: Diabetes is a major contributor to morbidity and mortality as well as the single most expensive health care condition in the world. Numerous interventions have attempted to improve control of this disorder and reduce its complications. Traditional care for diabetes centers on an individual clinician. More recently, recognition of the central role of the patient has come into vogue. Payors, including Medicare, now cover up to 13 hours of diabetes self-management and education programs annually. Patientcentered medical home efforts add an aspect of inclusiveness, but retain a medical focus and are being increasingly advocated and trained. To date, no research has focused on the use of interprofessional learning teams simultaneously delivering care and learning to work together.

Purpose: This project contributes to the development and training of interprofessional learner teams to enhance patient care. Intended outcomes include learner attitude and behavior changes and improvement in diabetic patients' biomarkers, empowerment and satisfaction.

Methods: Teams of 6-9 learners from eight fields and three universities work with cohorts of 6-21 African-American diabetic patients. The project includes team training, implementation of a diabetes self-management education (DSME) program and weekly telephone coaching. Learners complete the Centers for Disease Control and Prevention's TeamSTEPPS teamwork attitude questionnaire pre- and postintervention. Patients are recruited from the Aurora Midtown Clinic, which serves a largely Medicare/Medicaid population in Milwaukee's central city. Registries are searched for patients who fall out of quality goals; they are recruited by team members, with a target of 25 to 30 willing patients to complete the five DSME sessions and 4-week phone coaching around SMART goals. Patient biomarker data is tabulated, and pre- and postintervention Diabetes Empowerment Scale completed.

Results: Three cohorts of 42 patients and 23 students have completed the program. Patients demonstrated high attendance rates, improved diabetes knowledge and selfmanagement skills, and a trend in improvement in diabetes control compared to age/gender-matched controls. Students did not show a change in interprofessional attitude.

Conclusion: Challenges included coordinating schedules, demonstrating change of attitude in self-selected students, and discipline-specific supervision requirements. These programs demonstrated feasibility of concept for an interprofessional student-led DSME program to enhance patient care, with high student interest and engagement.

\section{A Meta-Analysis of Incidence and Risk Factors of Trastuzumab-Induced Cardiotoxicity in Breast Cancer}

Zeeshan Ali Jawa, Ruth M. Perez, Lydia Garlie, Maharaj Singh, Rubina Qamar, Bijoy K. Khandheria, Arshad Jahangir, Yang Shi

Internal Medicine, Medical College of Wisconsin; Aurora Research Institute, Aurora Health Care; University of Wisconsin-Madison; Medical Oncology, Aurora Health Care; Aurora Cardiovascular Services, Aurora Health Care; Sheikh Khalifa bin Hamad Al Thani Center for Integrative Research on Cardiovascular Aging, Aurora Health Care

Background: A monoclonal antibody, trastuzumab targets the human epidermal growth factor receptor 2 (HER2) 